\title{
Direct recognition of initiator elements by a component of the transcription factor IID complex
}

\author{
Jörg Kaufmann and Stephen T. Smale \\ Howard Hughes Medical Institute, Molecular Biology Institute, and Department of Microbiology and Immunology, \\ University of California, Los Angeles School of Medicine, Los Angeles, California 90024-1662 USA
}

\begin{abstract}
A core promoter element called an initiator (Inr) overlaps the transcription start site of numerous mammalian protein-coding genes. In promoters that lack a TATA box, the Inr is functionally analogous to TATA, in that it is capable of directing basal transcription by RNA polymerase II and of determining the precise site of transcription initiation. In promoters that contain a TATA box, the Inr can greatly enhance promoter strength. Mammalian Inr consensus sequences have been defined through functional studies and sequence comparisons of the start site regions of protein-coding genes. Here, we show that, in a DNase I footprinting assay with synthetic promoters, the purified TATA-binding protein complex TFIID specifically contacted the Inr. The TFIID-Inr interaction relies on the precise nucleotides needed for Inr function. Detection of the interaction was dependent either on a TATA box or on Sp1 bound to upstream sites. Furthermore, recombinant TFIIB appeared to influence the TFIID-Inr interaction, whereas TFIIA stabilized the TFIID-TATA interaction. These results demonstrate that distinct components of TFIID interact with the TATA boxes and Inr elements of core promoters for RNA polymerase II.
\end{abstract}

[Key Words: Transcription; TFIID; Inr; promoter; RNA polymerase II]

Received January 5, 1994; revised version accepted February 3, 1994.

Accurate transcription initiation from mammalian protein-coding genes depends on specific recognition of core promoter elements. For promoters that contain a TATA box, this recognition event is carried out by TFIID, a multiprotein complex containing a TATA-binding subunit called TBP and several additional factors known as TAFs (Zawel and Reinberg 1992; Hernandez 1993). For promoters that lack a TATA box, the proteins that functionally recognize the core control elements have not been clearly established. One class of TATA-lacking promoters includes those that contain an Inr element (Weis and Reinberg 1992; Kollmar and Farnham 1993; Smale 1994). An Inr can be defined as a basal control element that overlaps a transcription start site and is capable of determining the location of the start site in a promoter that lacks a TATA box (Smale and Baltimore 1989). Furthermore, an Inr can enhance the strength of a promoter that contains a TATA box, if the Inr is located $\sim 25 \mathrm{bp}$ downstream of TATA (Smale and Baltimore 1989; Smale et al. 1990).

Recently, through a functional analysis of 80 random and mutant Inr elements, we defined a loose consensus sequence for Inr activity in mammalian cells (Javahery et al. 1994). This consensus sequence, Py Py $A_{+1}$ N T/A Py $\mathrm{Py}$, is similar to a start site consensus that had been

${ }^{1}$ Corresponding author. defined solely through a sequence comparison of 500 genes transcribed by RNA polymerase II (Bucher 1990). Moreover, virtually every mammalian Inr that has been described matches this consensus (see Weis and Reinberg 1992; Kollmar and Farnham 1993; Smale 1994). Although a variety of proteins have been reported to bind to Inr elements in specific genes (Means and Farnham 1990; Roy et al. 1991; Seto et al. 1991; Du et al. 1993), binding of these proteins does not rely on the same nucleotides that are required for Inr activity (Kollmar and Farnham 1993; Javahery et al. 1994).

Two primary lines of evidence suggest that a component of the general transcription machinery, and specifically a component of the TFIID complex, may be responsible for Inr recognition. First, during in vitro transcription experiments, Inr activity typically has been observed in the presence of TFIID, but not in the presence of recombinant TBP (Smale et al. 1990; Conaway et al. 1991; Pugh and Tjian 1991; Wang and Van Dyke 1993; see Fig. 1A, below). Second, with some promoters that contain both TATA and Inr elements, DNase I footprinting experiments have revealed that TFIID contacts the DNA at the TATA box and also at sequences downstream of TATA (Sawadogo and Roeder 1985; Zhou et al. 1992; Purnell and Gilmour 1993). In two studies, mutations in an Inr element reduced the interaction between partially purified TFIID and the transcription start site 
region (Purnell and Gilmour 1993; Wang and Van Dyke 1993).

Despite the above evidence in support of a TFIID-Inr interaction, specific mutations in the functional Inr element of the adenovirus major late (AdML) promoter appear to have no effect on the binding of extensively purified TFIID (Chiang et al. 1993; J. Kaufmann and S.T. Smale, unpubl.). Instead, binding of purified TFIID to the AdML promoter appears to depend primarily on the TATA box and on sequences located upstream and/or downstream of the Inr (Chiang et al. 1993). This finding suggests that either (1) TFIID does not functionally interact with the Inr, or (2) in the AdML promoter, a specific interaction with the Inr is masked by the strong binding of TFID to surrounding sequences.

To analyze in more detail the role of TFID in Inr activity, we studied the binding of highly purified TFIID preparations to synthetic core promoters that contain a strong Inr but that lack the surrounding sequences found in the AdML promoter. Our results demonstrate that a component of TFID specifically interacts with the Inr, with the interaction depending on the precise sequences required for Inr function.

\section{Results}

The purified TFIID complex is required for Inr function

To investigate the possibility of a direct interaction between TFIID and Inr elements, we wished to analyze the binding of the purified protein complex to a core promoter containing a TATA box and an Inr but lacking the surrounding sequences that apparently contact TFIID in the AdML promoter. To this end, we studied a synthetic promoter containing only the AdML TATA box fused to the strong Inr from the terminal transferase (TdT) gene (plasmid 11634; see plasmid IV in Smale et al. 1990). For all of our experiments, TFIID was isolated from HeLa cells containing a retroviral insert expressing an epitopetagged TBP protein (Zhou et al. 1992). From nuclear extracts derived from these cells, TFIID could be purified to near homogeneity by phosphocellulose chromatography and immunoaffinity chromatography, using a monoclonal antibody that recognizes the amino-terminal epitope (Zhou et al. 1992). Figure 1B shows a silver-stained SDSpolyacrylamide gel that reveals the epitope-tagged TBP and the five largest TAFs (TAF250, TAF125, TAF95, TAF78, and TAF50; see Zhou et al. 1992). We expect that the smaller TAFs (see Zhou et al. 1992) were present in our preparations, although they could not be detected on the silver-stained gels.

Efficient transcription from the synthetic J1634 promoter was dependent on both the Inr element and on the intact TFIID complex (Fig. 1A). In a crude nuclear extract, the J1634 promoter was considerably stronger than a promoter containing a single base pair mutation in the $\operatorname{Inr}(J 3102,+3 G ;$ Fig. 1A, lanes 1,2). Transcription from both promoters was eliminated when the extract was depleted of TFIID by heat treatment (Nakajima et al. 1988) (lanes 3,4). Purified TFIID restored strong tran-

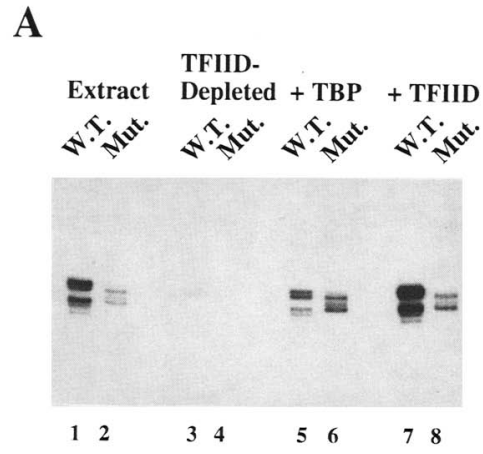

B

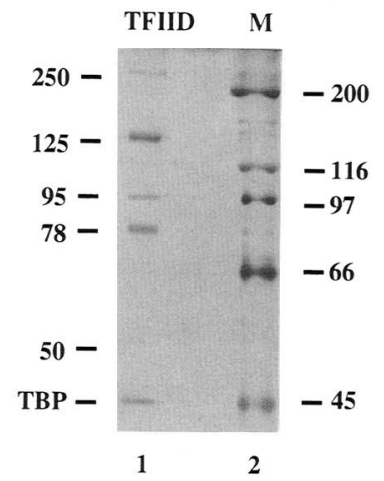

Figure 1. The intact TFIID complex is required for Inr activity. (A) In vitro transcription reactions were performed with $50 \mu \mathrm{g}$ of crude HeLa nuclear extract (lanes 1,2) or nuclear extract that had been depleted of TFIID activity by heat treatment (Nakajima et al. 1988, lanes 3-8). Reactions were supplemented with $5 \mu$ of human TBP expressed in E. coli or $5 \mu$ l of purified TFIID. Templates (300 ng) were either J1634 (TATA/Inr) or J3102 (Inr mutant, $+3 G$, see Fig. 3). (B) Purifed TFIID (30 $\mu$ l) (lane 1) was visualized on an SDS-polyacrylamide gel by silver staining. TFIID was purified as described (Zhou et al. 1992) from HeLa cells expressing an epitope-tagged TBP. Markers are shown in lane 2. The TAFs that are smaller than TBP (see Zhou et al. 1992) were not visible on the stained gel.

scription from the promoter containing the wild-type Inr and weaker transcription from the promoter containing the mutant Inr (lanes 7,8). However, recombinant human TBP expressed in Escherichia coli resulted in equivalent initiation frequencies from both promoters (lanes 5,61 , demonstrating that TBP is insufficient for Inr activity. A similar requirement for the TFIID complex has been found in studies of more complicated Inr-containing promoters and of promoters that depend on both an Inr and an upstream activator (Smale et al. 1990; Conaway et al. 1991; Pugh and Tiian 1991; Wang and Van Dyke 1993). However, in those experiments, the TAFs might have been needed for activity of the upstream activator but not for activity of the Inr. The promoters used here contain only TATA and Inr elements, confirming that the TFIID complex is essential for Inr activity.

\section{TFIID specifically interacts with the TdT Inr}

To test for binding of TFID to the strong TdT Inr, we 
performed DNase I footprinting experiments with the J1634 promoter. With the purified TFIID, we detected binding to the TATA box and weak protection of sequences downstream of TATA (Fig. 2B). Interestingly, a strong hypersensitive band was detected mapping to nucleotide +5 relative to the start site (Fig. 2B). This band is at a location that borders the $3^{\prime}$ end of the Inr. With recombinant TBP expressed in $E$. coli, strong protection of the TATA box was observed, but the hypersensitive site at +5 was absent (Fig. 2A). Furthermore, the hypersensitive site was strongly reduced when we tested a promoter containing a mutant TATA box (with the sequence GATATC) that was reported previously to reduce TATA activity by $\sim 100$-fold (Zenzie-Gregory et al. 1993) (Fig. 2C, lanes 10,11; see also Fig. 7, lane 12, below). A 2-bp mutation that abolished Inr activity $(-1 \mathrm{G}$,

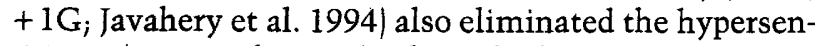
sitivity (Fig. 2C, lane 15). Thus, the hypersensitive site, but not the weak protection, appears to depend on the Inr element. (It should be noted that, in a given panel, all of the DNase footprinting probes were prepared by PCR with the same ${ }^{32}$ P-labeled primer, allowing all probes to possess the same specific activity.)

Although the transcribed strand in Figure $2 B$ primarily exhibited a hypersensitive site at the Inr, protection could be observed in DNase I footprinting experiments with a probe labeled on the nontranscribed strand. In Figure 2D, lane 18, weak protection was observed at the TATA box and at multiple locations downstream of TATA. Notably, two bands contained within the Inr were consistently protected relative to the bands both above and below the Inr. To achieve this result, it was necessary to concentrate the purified TFIID preparation threefold by centrifugation through a Centricon 30 concentrator (Amicon). The protection was reduced when a probe containing a 2-bp mutation in the Inr was tested (Fig. 2D, lane 22). Because this same mutation abolished the hypersensitive site observed on the transcribed strand (Fig. 2C, lane 15), the protection and the hypersensitive site most likely result from the same TFIID-Inr interaction. Taken together, the results in Figure 2 reveal an interaction between TFIID and the start site region that relies on sequence-specific contacts at both the -30 region and the Inr.

\section{The TFIID-Inr interaction depends on the precise nucleotides needed for Inr function}

The data presented above reveal a TFIID interaction at the Inr that may be required for Inr activity. An important criterion that must be met, however, is that the TFIID-Inr interaction and Inr activity must be depen-
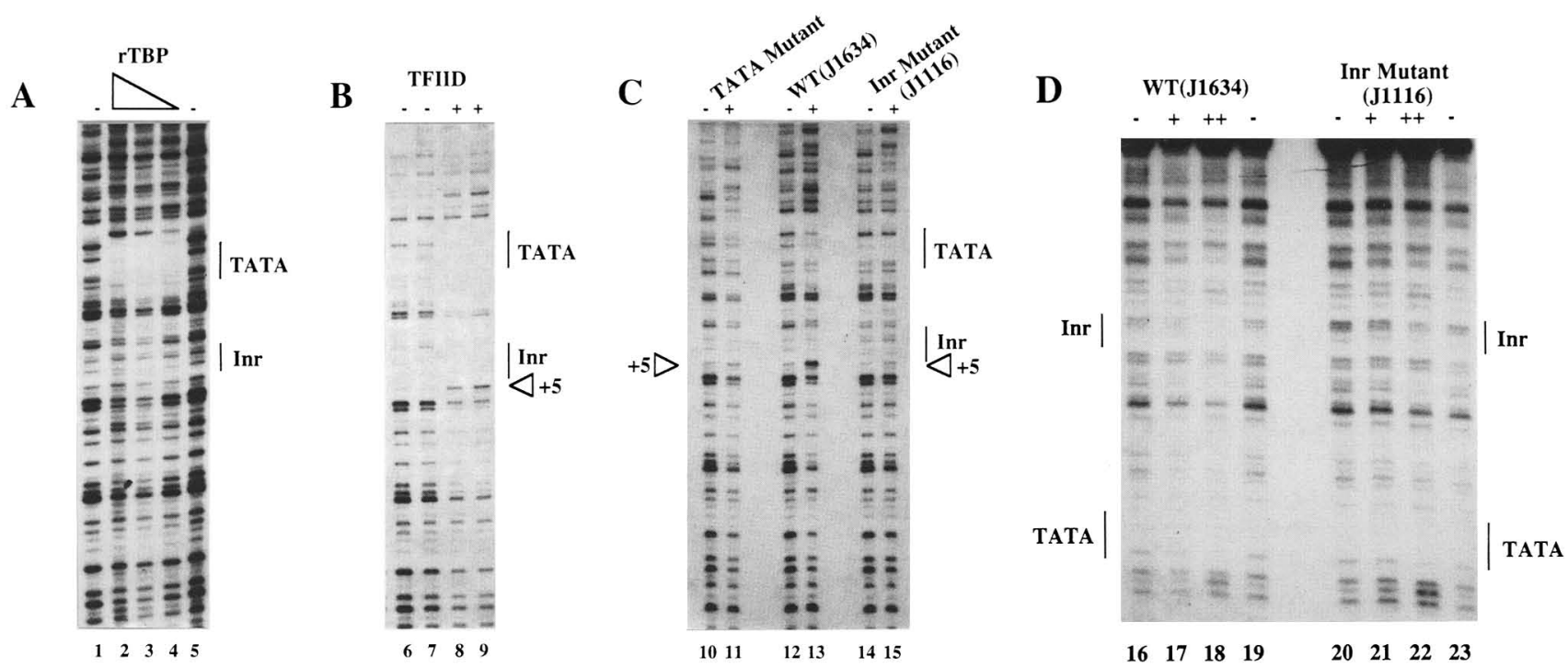

Figure 2. Purified TFIID contacts the Inr element in synthetic promoters containing the AdML TATA box and TdT Inr. $(A)$ Recombinant human TBP containing an epitope tag was partially purified from E. coli as described (Zhou et al. 1992). The footprinting probe, labeled on the transcribed strand, was prepared from plasmid J1634, containing the AdML TATA box and TdT Inr inserted into plasmid pSP72 (see plasmid IV in Smale et al. 1990). Reactions were performed in the absence (lanes 1,5) or presence (lanes 2-4) of decreasing amounts of TBP. The locations of the TATA and Inr are indicated at right. (B) DNase I footprint reactions were performed with J1634 in the absence (lanes 6,7$)$ and presence (lanes 8,9 ) of $7.5 \mu \mathrm{l}$ of purified TFIID. The locations of the TATA and Inr are indicated, as is the location of the strong hypersensitive site at $+5 .(C)$ DNase I footprinting reactions were performed in the absence $($ lanes $10,12,14)$ or presence (lanes 11,13,15) of $7.5 \mu$ l of purified TFIID, using plasmid J1634 (lanes 12,13), plasmid I (Smale et al. 1990; containing a deletion of the AdML TATA oligonucleotide, lanes 10,11), or plasmid J1116 (containing a 2-bp mutation, $-1 \mathrm{G},+1 \mathrm{G} ;$ lanes 14,15). The locations of the TATA box, Inr, and +5 hypersensitive site are indicated. $(D)$ DNase I footprinting reactions were performed with probes labeled on the nontranscribed strand (see Materials and methods) from plasmids J1634 (lanes 16-19) or J1116 (lanes 20-23). Reactions lack TFIID (lanes 16,19,20,23), contain $7.5 \mu$ l of purified TFIID (lanes 17,21), or contain $7.5 \mu l$ of TFIID after threefold concentration with a Centricon 30 concentrator (Amicon) (lanes 18,22). The locations of the TATA box and Inr element are indicated. 
dent on the same nucleotides. To address this issue, we analyzed nine mutant Inrs that had been tested previously for activity in the context of a promoter lacking a TATA box (Javahery et al. 1994). These mutants (Fig. 3, bottom) were inserted into a vector containing the AdML TATA box, but no upstream activator. In vitro transcription analyses with these plasmids (Fig. 3, lanes $1-10$ ) revealed Inr activities similar to those found in the context of the upstream Sp1 sites (Javahery et al. 1994), with only minor quantitative differences.

DNase I footprinting experiments with each of these mutant promoters revealed that the presence of the hypersensitive site correlates with Inr activity (Fig. 4). Mutants that eliminate activity (J3102, J1123, and J1116) did not exhibit hypersensitivity nor did random Inr elements that are largely inactive (J444 and J2549). (With these mutants, the weak TATA protection was also somewhat reduced, suggesting that one role of the TFIID-Inr interaction may be to stabilize binding of TBP to the TATA box.) In contrast, mutants with considerable Inr activity (J3521, J3121, and J2219) retained the hypersensitive site. (As noted by the question mark at the bottom of Figure 4 , we consider the results with the J2219 Inr to be inconclusive because although hypersensitivity was detected, the weak protection downstream of the hyper-

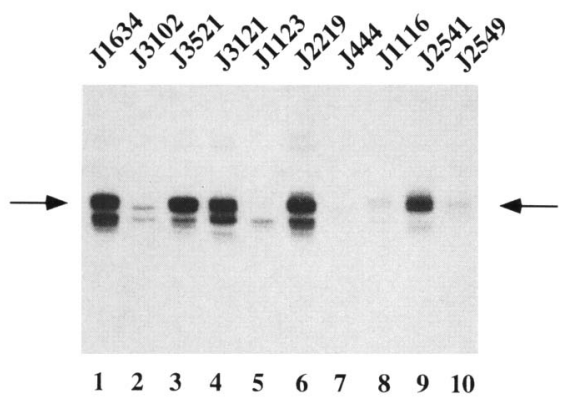

\begin{tabular}{|c|c|c|c|c|c|c|c|c|c|}
\hline J1634 & C C & C & $\mathbf{T}$ & C & & & $\mathbf{T}$ & C & $\mathbf{T}$ \\
\hline J3102 & $C \mathrm{C}$ & C & $T$ & C & A & $\mathbf{T}$ & $\underline{G}$ & C & $\mathrm{T}$ \\
\hline J3521 & A A & A & $\mathbf{T}$ & C & A & & $\mathrm{T}$ & C & $\mathbf{T}$ \\
\hline J3121 & C C & C & $\mathbf{T}$ & C & A & $\mathbf{T}$ & $\underline{\text { A }}$ & C & $\mathbf{T}$ \\
\hline J1123 & $C \mathrm{C}$ & C & $\mathbf{T}$ & C & I & & $\overline{\mathbf{T}}$ & $c$ & $\mathbf{T}$ \\
\hline J2219 & $\begin{array}{ll}C & C \\
& C\end{array}$ & C & $\mathrm{G}$ & & A & $\mathbf{T}$ & $T$ & & $T$ \\
\hline J1116 & C C & C & $\mathrm{T}$ & & & $T$ & $T$ & & $T$ \\
\hline J2541 & $\underline{\mathbf{T}} \mathbf{T}$ & C & $\underline{\mathrm{C}}$ & $\bar{C}$ & & $\mathbf{T}$ & $\mathrm{T}$ & $\underline{\mathbf{T}}$ & C \\
\hline 12540 & C C & C & $\underline{C}$ & C & A & $C$ & C & $\mathbf{T}$ & $\mathrm{T}$ \\
\hline
\end{tabular}

Figure 3. Functional activity of Inr mutants in the context of an upstream TATA box. In vitro transcription reactions were performed with $50 \mu \mathrm{g}$ of HeLa nuclear extract and $300 \mathrm{ng}$ of template DNA. Templates contained the AdML TATA box and either the TdT Inr (J1634, lane 1) or a mutant Inr (lanes 2-10). Inr sequences are shown at the bottom, with the nucleotides underlined that differ from the TdT Inr. The relative activities of the promoters (the mean of two independent experiments; $J 1634=100 \%$ ) are shown at the bottom of Fig. 4 . sensitive site was not observed as with the J1634 plasmid. We do not understand the significance of this weak downstream protection or whether it involves specific or nonspecific interactions.) A hypersensitive site was also detected at a similar location with the randomly-generated J254I Inr (see Javahery et al. 1994), even though the sequence of this active Inr is considerably different from the sequence of the TdT Inr (and therefore results in a very different DNase I digestion pattern in the absence of protein). The most striking comparison involves the two mutants that contain single base pair changes at the same +3 position. One of these mutants $(J 3102,+3 G)$ lacks Inr activity and hypersensitivity, whereas the other (J3121, +3A) retains both activity and hypersensitivity.

A gel mobility shift analysis was employed to confirm the correlation between Inr activity and TFIID binding. The gel shift experiments used agarose gel electrophoresis (see Materials and methods) and probes containing both the TATA and Inr elements. With the wild-type TdT Inr, a strong band was detected migrating at the expected location (Fig. 5, lane 6; Zhou et al. 1992). Mutation of the TATA box strongly reduced the TFIIDDNA complex (lane 3). A 2-bp mutation at -1 and +1 $(J 1116,-1 G,+1 G)$ or a single base pair mutation at +3 (J3102, +3G) reduced the intensity of the complex by two- to threefold (Fig. 5, lanes 9,12). Similar results were found in three independent experiments. These two Inr mutations strongly reduced Inr activity (see Fig. 3). Conversely, the mutation at +3 that retained Inr activity (J3121, +3A) resulted in no significant effect on the TFID-DNA complex (Fig. 5, lane 15). This result suggests that the binding of TFIID to the Inr increases the affinity of TFIID for a TATA-containing promoter. The mutant analysis using both DNase I footprinting and mobility retardation assays implicates TFIID as the only factor that has been identified whose interaction with the Inr depends on the precise sequences required for Inr activity.

\section{TFIIA and TFIIB have differential influences on TFIID binding to TATA and Inr elements}

Two general transcription factors known to interact with TFIID are TFIIA and TFIIB. In some assays, TFIIA stabilizes the binding of TBP to TATA boxes (Ranish et al. 1992; Zawel and Reinberg 1992). TFIIB has been shown to interact both with TBP and with Drosophila TAF40 (Buratowski et al. 1989; Zawel and Reinberg 1992; Goodrich et al. 1993). Moreover, TFIIB induced an interaction with the start site region of the AdML promoter when added to a binding reaction that contained TFIID (Buratowski et al. 1989; Maldonado et al. 1990; Moncollin et al. 1992). To analyze the effect of TFIIA and TFIIB on the specific interaction between TFIID and the Inr element, we performed the experiments shown in Figure 6. For these experiments we began with a lower concentration of TFIID that when added alone is not sufficient to reveal interactions with the core promoter (Fig. 6, lanes 2,7,11). These conditions were found to be 

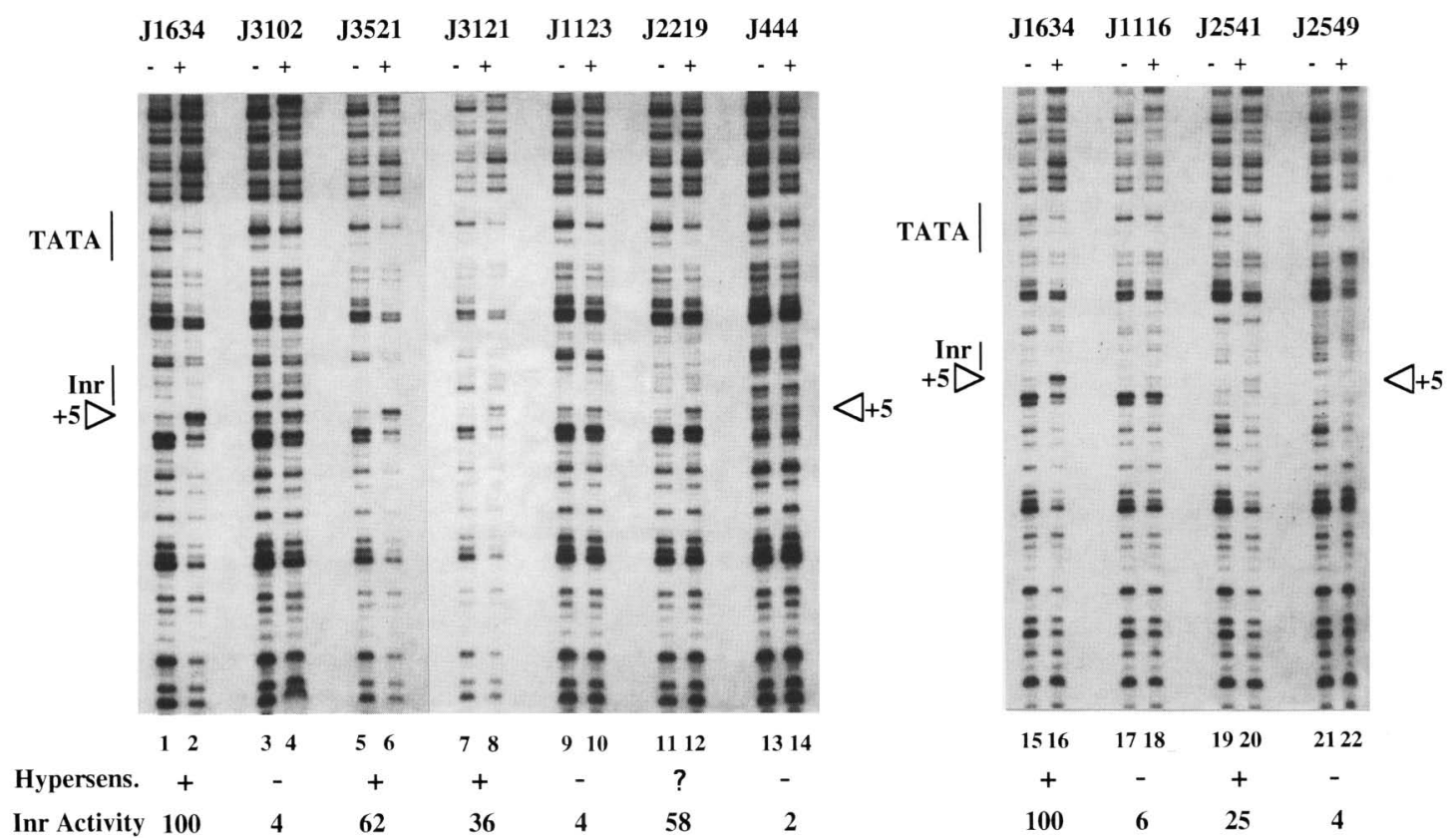

Figure 4. The TFIID-Inr interaction and Inr activity depend on the same sequences. DNase I footprinting experiments were performed with $5000 \mathrm{cpm}$ of ${ }^{32} \mathrm{P}$-labeled probes (see Fig. 2) containing wild-type and mutant promoters. All probes were prepared with the same labeled primer, resulting in similar specific activities. Reactions were performed in the absence (odd-numbered lanes) or presence (even- numbered lanes) of $7.5 \mu \mathrm{l}$ of purified TFIID. The location of the +5 hypersensitive site is indicated.

the most effective for revealing influences on the binding of TFIID.

Figure 6 (lanes 3,4) shows that recombinant yeast TFIIA (Ranish et al. 1992) strongly induces binding of

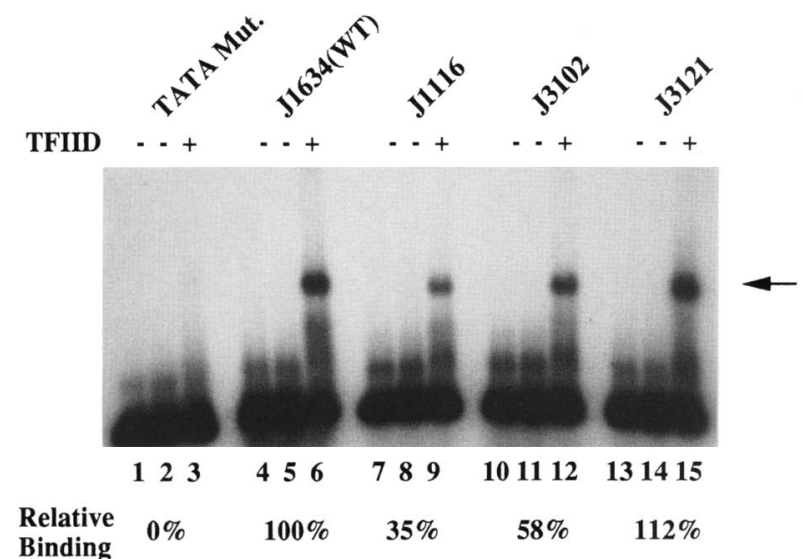

Figure 5. The TFIID-Inr interaction enhances TFIID binding in a mobility retardation assay. This assay was performed using agarose gel electrophoresis as described (see Materials and methods). Probes were derived from plasmid I (lanes 1-3, see Smale et al. 1990), J1634 (lanes 4-6), J1116 (lanes 7-9), J3102 (lanes 10-12), and J3121 (lanes 13-15). Binding conditions were the same as for DNase I footprinting experiments and lacked (lanes $1,2,4,5,7,8,10,11,13,14$ ) or contained $7.5 \mu \mathrm{l}$ of purified TFIID. Quantitation was performed by Phosphorlmager analysis (Molecular Dynamics). The results are representative of three independent experiments. The arrow indicates the specific protein-DNA complex.
TFIID to the TATA box. However, the TFIIA did not induce the hypersensitive site at the Inr. Thus, increasing the affinity of TFIID for the TATA box does not appear to enhance the binding of TFIID to the Inr. (In Fig. 6 , lanes $3-5$, the TFIIA appears to result in nonspecific protection of the probe; this protection most likely results from contaminating proteins in the partially purified TFIIA preparation.)

Interestingly, recombinant human TFIIB $(\mathrm{Ha}$ et al. 1991) influenced the interactions taking place near the Inr but had little influence on TFIID binding to the TATA box (Fig. 6, lanes 8,9). As expected, TFIIB did not induce the hypersensitive site in the presence of recombinant TBP (data not shown). In Figure 6, TFIIB resulted in a modest hypersensitivity of the +5 band relative to that observed with TFIID alone (cf. lanes 7 and 9). However, the most striking effect of TFIIB was to induce protection of sequences downstream of the Inr (lane 9). Thus, although TFIIB may stabilize the binding of TFIID to the Inr and downstream sequences, an alternative explanation is that TFIIB itself may interact with sequences near the Inr in a TFIID-dependent manner.

With both TFIIA and TFIIB added to the binding reaction, clear interactions were detected at the TATA box and at the Inr (Fig. 6, lane 13). However, despite the efficient protection of the TATA box and the strong hypersensitive site at the Inr, the Inr remained only weakly protected on the transcribed strand. Analysis of the Inr mutants again revealed that the TFIID-Inr interaction correlates with the sequence requirements for activity. Most strikingly, the $3121(+3 \mathrm{~A})$ mutant retained strong hypersensitivity, but only a weak interaction was de- 
Figure 6. TFIIA and TFIIB differentially influence the interaction between TFIID and synthetic promoters. DNase I footprinting experiments were performed with the J1634 promoter (lanes $1-13$ ) or the Inr mutants J3102 (lanes 14,15), J3521 (lanes 16,17 ), or J3121 (lanes 18,19 ). Reactions contained no protein (lanes 1,6,12,14, 16,18 ), $2.5 \mu \mathrm{l}$ of purified TFIID (lanes 2-4, $7-9,11,13,15,17,19$ ), 0.25 (lane 3 ) or 2.5 (lanes $4,5,13,15,17,19$ ) $\mu$ l of partially purified recombinant yeast TFIIA expressed in E. coli (Ranish et al. 1992), and 0.25 (lane 8) or 2.5 (lanes $9,10,13,15,17,19) \mu$ l of partially purified recombinant TFIIB (Ha et al. 1991). The locations of the TATA box, Inr, and +5 hypersensitive site are indicated. (Bottom) The relative promoter strengths, as determined from Fig. 3, are shown.

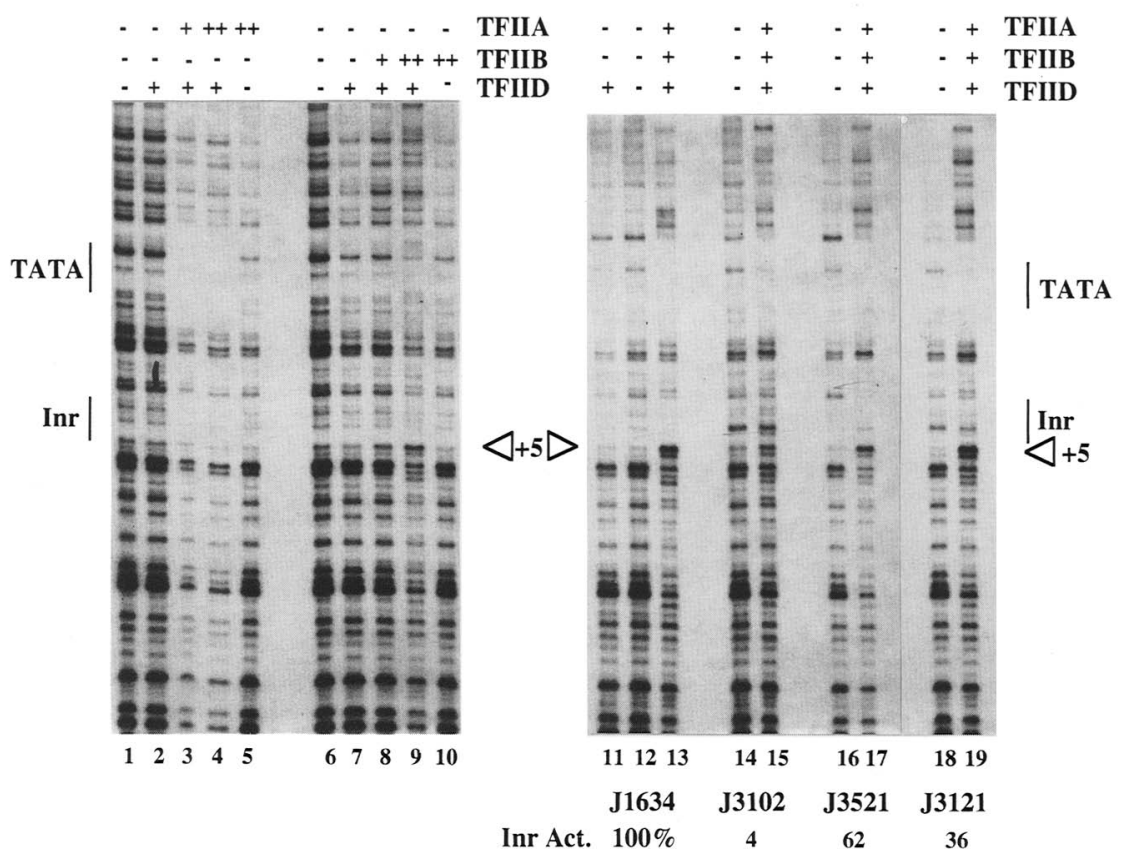

tected with the $3102(+3 G)$ mutant (Fig. 6, lanes 14-19). In the presence of TFIIA and TFIIB, a weak interaction, similar to that found with $J 3102$, was observed with all the Inr elements that are functionally inactive /data not shown). These results confirm that the specific interaction between TFIID and the Inr correlates with Inr activity.

In the presence of Sp1, TFIID interacts with the Inr in the absence of a TATA box

The experiments described above demonstrate that a component of TFIID specifically interacts with the Inr in a promoter that contains a TATA box, but little evidence of the interaction was detected in the absence of a TATA box (see Fig. 2C, lanes 10,11). Figure 7, lane 12, shows the best interaction between TFIID and the isolated TdT Inr that we detected after numerous attempts. In this reaction, a weak hypersensitive site is visible at the +5 position and protection is visible downstream of the Inr. However, the ratio between the bands at +5 and +8 is not altered dramatically, as it is in the presence of a TATA box (cf. Fig. 7, lane 12, with Fig. 2B, lane 8, and Fig. 4, lane 2). In functional assays, transcription directed
Figure 7. Spl stimulates the interaction between TFIID and the Inr. DNase I footprinting reactions were performed as in Fig. 2, except the total volume was increased to $45 \mu$ l. Plasmid VI (Smale et al. 1990; lanes 1-5) contains six Spl-binding sites upstream of the TdT Inr, in the absence of a functional TATA box. R3102 (Javahery et al. 1994; lanes 6-9) is similar to plasmid VI, except it contains a single base pair change ( $+3 G)$ that abolished Inr activity. Plasmid I (Smale et al. 1990; lanes 10-14) contains the isolated TdT Inr inserted into the pSP72 vector. Plasmid VII (Smale et al. 1990, lanes 15-19) contains six Spl-binding sites upstream of a core promoter containing both the AdML TATA box and the TdT Inr. Reactions were performed in the absence of added proteins (lanes $1,4,6,9,10,14,15,19$ ), in the

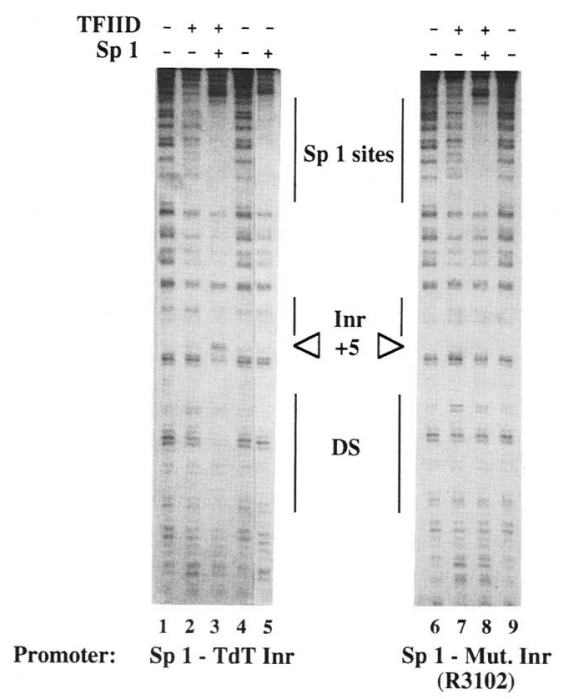

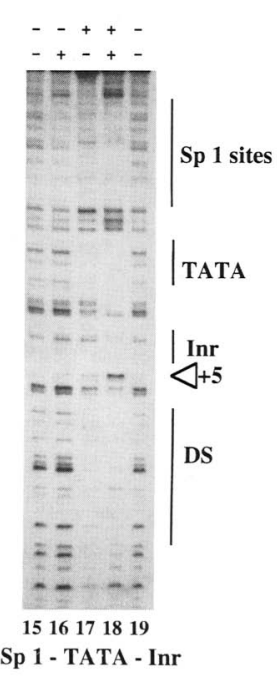

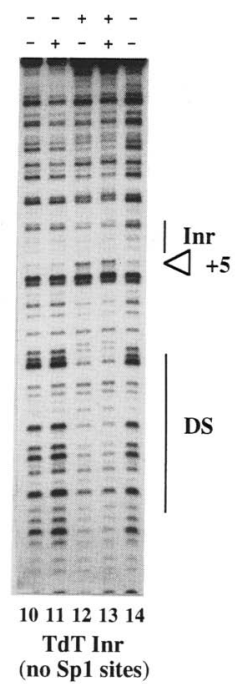

Sp 1 - TATA - In

presence of $7.5 \mu \mathrm{l}$ of TFIID (lanes 2,3,7,8,12,13) or $3 \mu \mathrm{l}$ of TFID (lanes 17,18), and in the presence of $2 \mu \mathrm{l}$ of affinity-purified Sp1 (Promega; lanes $3,5,8,11,13,16,18$ ). The locations of the Sp1 sites, the TATA box, Inr, the +5 hypersensitive site, and the downstream protection (DS) are indicated. The first and second panels were derived from the same experiment, but the third and fourth panels were from separate experiments. 
by the isolated Inr is extremely weak but can be greatly enhanced by the binding of Spl to upstream sites (Smale and Baltimore 1989; Smale et al. 1990). Therefore, to determine whether TFIID is capable of interacting with the Inr in the absence of a TATA box, we performed DNase I footprinting experiments in the presence of TFIID and purified Spl.

With a synthetic promoter containing six Spl-binding sites upstream of both TATA and Inr elements, TFIID bound to the TATA box and to sequences downstream of the Inr in the absence of Spl (Fig. 7, lane 17). In this experiment (lanes 17 and 18), the hypersensitive site at +5 was very weak because a smaller amount of TFIID was added relative to the amounts used in Figures 2,4, and 7 (lanes 1-15). With both Spl and TFID added to the reaction, the hypersensitive site was enhanced (lane 18). Similar effects of activator proteins on the binding of TFIID to TATA-containing promoters have been reported (e.g., Horikoshi et al. 1988).

In another promoter, the TATA box was mutated to a sequence that reduces TATA activity by $\sim 100$-fold (TATAAA to GATATC; Zenzie-Gregory et al. 1993). With this promoter, no specific interactions were observed at the core promoter with TFIID in the absence of Spl (Fig. 7, lane 2). However, in the presence of Sp1, a clear interaction was detected at the Inr and protection was observed downstream of the Inr (lane 3). Spl did not induce the binding of TFIID to a promoter containing a point mutant at the Inr (lane 8) or to the promoter containing the isolated Inr (cf. lanes 12 and 13). These results demonstrate that the specific interaction between TFIID and an Inr can occur in the absence of a functional TATA box.

\section{Discussion}

The data presented in this manuscript demonstrate that a component of the TFIID complex is involved in Inr activity. In vitro transcription experiments with promoters containing only a TATA box and an Inr element confirmed that Inr activity requires the intact TFIID complex. DNase I footprinting studies then revealed that purified TFIID directly contacts the Inr. The TFIID-Inr interaction depended on the same nucleotides that were found in an extensive mutant analysis to be required for Inr function. The relevance of the interaction was further supported by experiments suggesting that the general transcription factor TFIIB specifically influences the interaction at the Inr. Finally, we found that TFIID can contact the Inr in a promoter that lacks a functional TATA box, primarily if $\mathrm{Spl}$ is bound to upstream sites.

In our experiments the TFIID-Inr interaction was revealed most clearly by the presence of a strong hypersensitive site in the DNase I footprinting experiments with a probe labeled on the transcribed strand. The absence of strong protection of the Inr partially results from the fact that the TFIID purified by immunoaffinity chromatography is not highly concentrated. However, the same protein preparations are concentrated enough to give strong protection of the TATA box (Figs. 6 and 7) and of sequences downstream of the start site in the AdML promoter (data not shown). Therefore, it appears that a low affinity of the TFIID-Inr interaction also contributes to the absence of protection. The loose consensus sequence for Inr activity (Javahery et al. 1994) previously suggested to us that the functional Inr-binding protein would interact with a relatively low affinity. If the functional protein bound independently and with high affinity, this protein would be capable of tightly interacting with the genome at an enormous number of sequences that match the consensus but are not associated with promoter regions. Another reason why we might expect a low-affinity interaction concerns the events that must occur in the vicinity of the Inr after the initial recognition event. Subsequent to this initial recognition, RNA polymerase II must become associated with the start site region and the DNA must unwind to allow the initiation of RNA synthesis. If a high-affinity interaction occurs, it would most likely block transcription initiation.

The possible stabilization of the TFIID-Inr interaction by TFIIB is consistent with results published previously (Buratowski et al. 1989; Maldonado et al. 1990; Moncollin et al. 1992). In particular, one study with purified components revealed protection of the AdML start site region in a DNase I footprinting reaction containing both TFIIB and the TFIID complex (Maldonado et al. 1990). In those experiments, it was not clear whether TFIID or TFIIB interacted directly with the start site or whether the interaction was dependent on an Inr. Recently, TFIIB has been shown to interact with Drosophila TAF40 (Goodrich et al. 1993), suggesting that this protein-protein interaction may enhance the binding of TFIID to the Inr and, furthermore, that Drosophila TAF40 and the human homolog of this TAF may be the Inr-binding subunit of TFIID.

In the absence of a TATA box, TFIID bound to the Inr primarily when Spl was bound to upstream sites. Spl interacts directly with Drosophila TAF110 (Hoey et al. 1993|, but we have not established that Spl induces the TFIID-Inr interaction by directly binding to this TAF. For the purposes of this study, the use of $\mathrm{Sp} 1$ allowed us to confirm that TFID is capable of interacting with an Inr in the absence of a TATA box.

It may be noteworthy that with the AdML promoter and with the synthetic promoter shown in Figure 7, TFIID appears to bind more tightly to the sequences surrounding the Inr than to the Inr itself (Fig. 7, lanes 15-19; Chiang et al. 1993; J. Kaufmann and S.T. Smale, unpubl.). In contrast, the Inr enhances promoter strength much more strongly than do the surrounding sequences (data not shown). This observation suggests that the strength of a core promoter does not depend simply on the overall affinity of the TFIID complex. Instead, the interaction between TFIID and the Inr is likely to play an important mechanistic role during the initiation reaction. Possibly, the TFIID-Inr interaction aids in the recruitment of TFIIB to the transcription start site, as suggested by the data in Figure 6. Alternatively, because the TFIID-Inr interaction occurs at the actual site of initia- 
tion, it may play a direct role in template unwinding or in the recruitment and positioning of RNA polymerase II.

Although the human TFIID used in our experiments appears to bind very weakly to the Inr and more strongly to surrounding sequences in promoters like AdML (Chiang et al. 1993; J. Kaufmann and S.T. Smale, unpubl.), Drosophila TFIID appears to contact the consensus Inr in the Drosophila hsp 70 promoter much more efficiently (Purnell and Gilmour 1993; D. Gilmour, pers. comm.). Moreover, the interaction of TFIID with the hsp70 Inr does not appear to be obscured by interactions between TFIID and surrounding sequences as in the AdML promoter (Purnell and Gilmour 1993). The stronger TFIID-Inr interaction observed in the Drosophila system may reflect the fact that the Drosophila Inr consensus sequence, although similar to the mammalian consensus, appears to be more restrictive (Cherbas and Cherbas 1993). This restrictive consensus sequence may be needed for TFIID to bind with higher affinity and with specificity within the Drosophila genome.

Several other proteins have been reported as specific Inr-binding proteins, including E2F(HIP1), TFII-I, USF, and YY1 (Means and Farnham 1990; Roy et al. 1991; Seto et al. 1991; Du et al. 1993). However, the binding of these proteins does not depend on the precise sequences required for Inr activity (Javahery et al. 1994), making it highly unlikely that the binding detected in our experiments results from these proteins contaminating our TFIID preparations. Instead, we suggest that these proteins act as auxiliary activators that stimulate transcription through gene-specific Inr elements. It remains to be determined whether the elements recognized by any of these other proteins can carry out Inr activity in a core promoter that lacks a TFIID recognition site at either the -30 region or the start site.

A previous study by Carcamo et al. (1991) demonstrated that purified RNA polymerase II activates transcription from CA dinucleotides, in the absence of TFIID and other general transcription factors. Our results do not exclude the possibility that in addition to recognition of the Inr by TFIID, RNA polymerase II may also prefer to begin RNA synthesis from CA dinucleotides. In support of this idea, a comparison of 500 promoters (Bucher 1990) found a strong preference for a $\mathrm{C}$ at the -1 position, yet we found no strong preference for a $\mathrm{C}$ (vs. a T) when we defined the functional Inr consensus sequence (Javahery et al. 1994). Possibly, the prevalence of a $\mathrm{C}$ at that position reflects a preference by RNA polymerase II during the actual initiation of RNA synthesis. Thus, the consensus Inr derived through sequence comparisons (Bucher 1990) may contain recognition sites for both TFIID and RNA polymerase II.

\section{Materials and methods}

\section{Protein preparation}

The epitope-tagged TFIID complex was isolated from LTR $\alpha 3$ cells by affinity purification as described (Zhou et al. 1992).
Preparation of the partially purified epitope-tagged TBP protein from E. coli was as described previously (Zhou et al. 1992). For the experiment in Figure 2D, the TFIID was concentrated threefold by centrifugation through a Centricon 30 concentrator (Amicon). Partially purified recombinant yeast TFIIA and human TFIIB expressed in E. coli were kindly provided by Dr. Michael Carey (University of California, Los Angeles). The human Spl was affinity purified (Promega).

\section{In vitro transcription assays}

In vitro transcription reactions were performed as described (Smale and Baltimore 1989) with $300 \mathrm{ng}$ of template DNA and $50 \mu \mathrm{g}$ of crude HeLa nuclear extract or nuclear extract that had been depleted of TFIID activity by heat treatment (Nakajima et al. 1988). RNA products were purified and analyzed by primer extension analysis using a ${ }^{32}$ P-labeled SP6 promoter primer (Promega; see Smale et al. 1990). cDNA products were visualized by electrophoresis on an $8 \%$ denaturing polyacrylamide gel, followed by autoradiography. Quantitation of signals was determined by PhosphorImager analysis (Molecular Dynamics).

\section{DNA-binding assays}

DNA probes were prepared by PCR using a $5^{\prime}$ end-labeled SP6 primer (Promega), complementary to sequences downstream of the AdML promoter, and an unlabeled primer (5'-GGCCGATTCATTAATGCAGG-3'), complementary to sequences upstream of the promoter. For Figure 2D, the opposite primer was labeled and PCR was performed with an unlabeled SP6 primer. Binding reactions for DNase I footprinting experiments were performed for $30 \mathrm{~min}$ at $30^{\circ} \mathrm{C}$ in a total volume of $15 \mu \mathrm{l}$, containing $5000 \mathrm{cpm}$ of ${ }^{32} \mathrm{P}$-labeled probe, $10 \mathrm{mM} \mathrm{MgCl}_{2}, 2 \mu \mathrm{g}$ of BSA, $1 \mathrm{mM}$ DTT, $10 \mathrm{~mm}$ HEPES (pH 7.9), 10\% glycerol, $0.1 \mathrm{~mm}$ EDTA, and $50 \mathrm{mM} \mathrm{KCl}$. Nonspecific competitor DNA was not added to the reactions. The labeled probes were then partially digested with DNase I, and cleaved DNA molecules were purified and analyzed by electrophoresis on an $8 \%$ denaturing polyacrylamide gel (see Lo et al. 1991).

Mobility retardation experiments were performed using agarose gel electrophoresis by the method described (Zhou et al. 1992), with a modified running buffer described by P. Lieberman and A. Berk (in prep.). Labeled probes for mobility retardation experiments were prepared by the same method as those used for DNase I footprinting experiments. Binding conditions were the same as above, except $10,000 \mathrm{cpm}$ of each ${ }^{32} \mathrm{P}$-labeled probe was used. Signals were quantitated by PhosphorImager analysis.

\section{Acknowledgments}

We thank Qiang Zhou, Paul Lieberman, and Arnold Berk for assistance with the TFIID purification and gel shift experiments, Michael Carey for providing recombinant TFIIA and TFIIB, and John Lee for plasmids containing mutant Inrs. We are also grateful to Arnold Berk and Kiersten Lo for reading the manuscript and to David Gilmour for helpful discussions and communication of results prior to their publication.

The publication costs of this article were defrayed in part by payment of page charges. This article must therefore be hereby marked "advertisement" in accordance with 18 USC section 1734 solely to indicate this fact.

\section{References}

Bucher, P. 1990. Weight matrix descriptions of four eukaryotic RNA polymerase II promoter elements derived from 502 unrelated promoter sequences. J. Mol. Biol. 212: 563-578. 
Buratowski, S., S. Hahn, L. Guarente, and P.A. Sharp. 1989. Five intermediate complexes in transcription initiation by RNA polymerase II. Cell 56: 549-561.

Carcamo, J., L. Buckbinder, and D. Reinberg. 1991. The initiator directs the assembly of a transcription factor IID-dependent transcription complex. Proc. Natl. Acad. Sci. 88: 8052-8056.

Cherbas, L. and P. Cherbas. 1993. The arthropod initiator: The capsite consensus plays an important role in transcription. Insect Biochem. Mol. Biol. 23: 81-90.

Chiang, C.-M., H. Ge, Z. Wang, A. Hoffmann, and R.G. Roeder. 1993. Unique TATA-binding protein containing complexes and cofactors involved in transcription by RNA polymerases II and III. EMBO J. 12: 2749-2762.

Conaway, J.W., J.P. Hanley, K.P. Garrett, and R.C. Conaway. 1991. Transcription initiated by RNA polymerase II and transcription factors from liver: Structure and action of transcription factors $\epsilon$ and $\tau$. J. Biol. Chem. 266: 7804-7811.

Du, H., A.L. Roy, and R.G. Roeder. 1993. Human transcription factor USF stimulates transcription through initiator elements of the HIV-1 and the Ad-ML promoters. EMBO $J$. 12: $501-511$.

Goodrich, J.A., T. Hoey, C.J. Thut, A. Admon, and R. Tjian. 1993. Drosophila $\mathrm{TAF}_{\mathrm{II}} 40$ interacts with both a VP16 activation domain and the basal transcription factor TFIIB. Cell 75: 519-530.

Ha, I., W.S. Lane, and D. Reinberg. 1991. Cloning of a human gene encoding the general transcription initiation factor IIB. Nature 352: 689-695.

Hernandez, N. 1993. TBP, a universal eukaryotic transcription factor? Genes \& Dev. 7: 1291-1308.

Hoey, T., R.O. Weinzierl, G. Gill, J.L. Chen, B.D. Dynlacht, and R. Tjian. 1993. Molecular cloning and functional analysis of Drosophila TAF110 reveal properties expected of a coactivator. Cell 72: 247-260.

Horikoshi, M., T. Hai, Y.S. Lin, M.R. Green, and R.G. Roeder. 1988. Transcription factor ATF interacts with the TATA factor to facilitate establishment of a preinitiation complex. Cell 54: 1033-1042.

Javahery, R., A. Khachi, K. Lo, B. Zenzie-Gregory, and S.T. Smale. 1994. DNA sequence requirements for transcriptional initiator activity in mammalian cells. Mol. Cell. Biol. 14: (in press).

Kollmar, R. and P.J. Farnham. 1993. Site-specific initiation of transcription by RNA polymerase II. Proc. Soc. Exp. Biol. Med. 203: 127-139.

Lo, K., N.R. Landau, and S.T. Smale. 1991. LyF-1, a transcriptional regulator that interacts with a novel class of promoters for lymphocyte-specific genes. Mol. Cell. Biol. 11: 52295243.

Maldonado, E., I. Ha, P. Cortes, L. Weis, and D. Reinberg. 1990. Factors involved in specific transcription by mammalian RNA polymerase II: Role of transcription factors IIA, IID, and IIB during formation of a transcription-competent complex. Mol. Cell. Biol. 10: 6335-6347.

Means, A.L. and P.J. Farnham. 1990. Transcription initiation from the dihydrofolate reductase promoter is positioned by HIPl binding at the initiation site. Mol. Cell. Biol. 10: 653661.

Moncollin, V., L. Fischer, B. Cavallini, J.-M. Egly, and P. Chambon. 1992. Class II (B) general transcription factor (TFIIB) that binds to the template-committed preinitiation complex is different from general transcription factor BTF3. Proc. Natl. Acad. Sci. 89: 397-401.

Nakajima, N., M. Horikoshi, and R.G. Roeder. 1988. Factors involved in specific transcription by mammalian RNA polymerase II: Purification, genetic specificity, and TATA box- promoter interactions of TFIID. Mol. Cell. Biol. 8: 40284040.

Pugh, B.F. and R. Tjian. 1991. Transcription from a TATA-less promoter requires a multisubunit TFIID complex. Genes \& Dev. 5: 1935-1945.

Purnell, B.A. and D.S. Gilmour. 1993. Contribution of sequences downstream of the TATA element to a proteinDNA complex containing the TATA-binding protein. Mol. Cell. Biol. 13: 2593-2603.

Ranish, J.A., W.S. Lane, and S. Hahn. 1992. Isolation of two genes that encode subunits of the yeast transcription factor IIA. Science 255: 1227-1129.

Roy, A.L., M. Meisterernst, P. Pognonec, and R.G. Roeder. 1991. Cooperative interaction of an initiator-binding transcription initiation factor and the helix-loop-helix activator USF. $\mathrm{Na}$ ture 354: 245-248.

Sawadogo, M. and R.G. Roeder. 1985. Interaction of a genespecific transcription factor with the adenovirus major late promoter of the TATA box region. Cell 43: 165-175.

Seto, E., Y. Shi, and T. Shenk. 1991. YY1 is an initiator sequence-binding protein that directs and activates transcription in vitro. Nature $354: 241-245$.

Smale, S.T. 1994. Architecture of core promoters for eukaryotic protein-coding genes. In Transcription: Mechanisms and regulation (ed. R.C. Conaway and J.W. Conaway), pp. 63-81. Raven Press, New York.

Smale, S.T. and D. Baltimore. 1989. The "initiator" as a transcription control element. Cell 57: 103-113.

Smale, S.T., M.C. Schmidt, A.J. Berk, and D. Baltimore. 1990. Transcriptional activation by $\mathrm{Spl}$ as directed through TATA or initiator: Specific requirement for mammalian transcription factor IID. Proc. Natl. Acad. Sci. 87: 4509-4513.

Wang, J.C. and M.W. Van Dyke. 1993. Initiator sequences direct downstream promoter binding by human transcription factor IID. Biochim. Biophys. Acta 1216: 73-80.

Weis, L. and D. Reinberg. 1992. Transcription by RNA polymerase II: Initiator-directed formation of transcription-competent complexes. FASEB J. 6: 3300-3309.

Zawel, L. and D. Reinberg. 1992. Advances in RNA polymerase II transcription. Curr. Opin. Cell Biol. 4: 488-495.

Zenzie-Gregory, B., A. Khachi, I.P. Garraway, and S.T. Smale. 1993. Mechanism of initiator-mediated transcription: Evidence for a functional interaction between the TATA-binding protein and DNA in the absence of a specific recognition sequence. Mol. Cell. Biol. 13: 3841-3849.

Zhou, Q., P.M. Lieberman, T.G. Boyer, and A.J. Berk. 1992. Holo-TFIID supports transcriptional stimulation by diverse activators and from a TATA-less promoter. Genes \& Dev. 6: 1964-1974. 


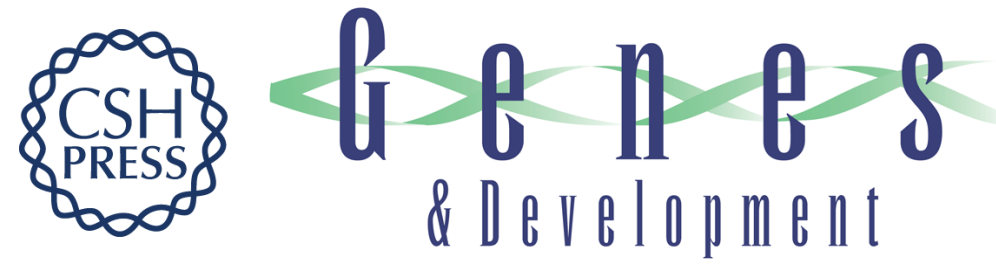

\section{Direct recognition of initiator elements by a component of the transcription factor IID complex.}

J Kaufmann and S T Smale

Genes Dev. 1994, 8:

Access the most recent version at doi:10.1101/gad.8.7.821

References This article cites 30 articles, 13 of which can be accessed free at: http://genesdev.cshlp.org/content/8/7/821.full.html\#ref-list-1

License

Email Alerting Service

Receive free email alerts when new articles cite this article - sign up in the box at the top right corner of the article or click here.

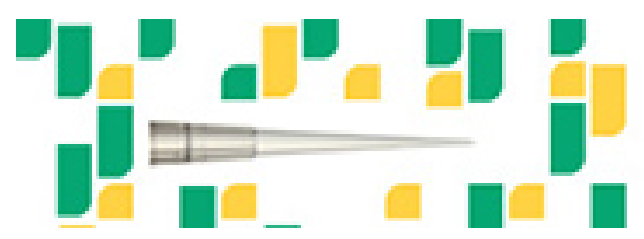

Focused on your science.

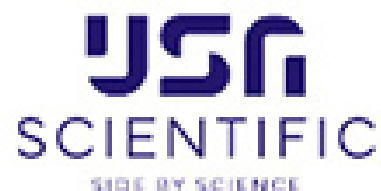

Copyright @ Cold Spring Harbor Laboratory Press 